\title{
Análisis de resultados comparativos en el aprendizaje de la Programación en Ingeniería de Sistemas a partir de tres modelos de trabajo en grupo ${ }^{1}$
}

\section{Analysis of comparative results in the learning of Programming in Systems Engineering from three models of group work}

\author{
O. I. Trejos
}

Recibido: febrero 15 de 2019 - Aceptado: septiembre 30 de 2019

\begin{abstract}
Resumen-- Esta investigación busca proporcionar elementos de juicio al docente para determinar cuál podría ser el modelo de trabajo en grupo apropiado en programación de computadores en Ingeniería. Se adoptó una estrategia de trabajos en grupo durante 6 semestres acudiendo a tres modelos: modelo 4Q, modelo de talento académico y modelo de agrupamiento aleatorio. Posteriormente se hicieron las comparaciones respectivas basadas en los resultados obtenidos. Éstos permiten entender algunas de las interacciones de cada grupo y la dinámica que, a su interior, se van dando. Se concluyó que es posible escoger un modelo de conformación de grupos de trabajo en programación de computadores en Ingeniería de Sistemas si se perfila apropiadamente el grupo de estudiantes.

Palabras clave-- Aprendizaje, ingeniería de sistemas, modelo $4 Q$, modelo aleatorio, programación de computadores, trabajo en grupo.
\end{abstract}

Abstract-- The target of this research is to find judge elements, using experiments in the classroom, where teachers could know the excellence model to conform effective teamworks for learning computer programming in the first programming

${ }^{1}$ Este artículo es producto del proyecto de investigación 6-15-10 (modalidad sin financiación) titulado "Desarrollo de un modelo metodológico para aprender Programación en Ingeniería de Sistemas basado en aprendizaje significativo y el modelo $4 Q$ de preferencias de pensamiento" aprobado por el Consejo de Facultad de Ingenierías y registrado por la Vicerrectoría de Investigaciones, Innovación y Extensión de la Universidad Tecnológica de Pereira. Línea de investigación: Nuevas metodologías de aprendizaje en formación tecnológica y científica, Grupo de Investigación en Informática.

O. I. Trejos, Universidad Tecnológica de Pereira, Pereira, Colombia, email: omartrejos@utp.edu.co.

Como citar este artículo: Trejos, I. O. Análisis de resultados comparativos en el aprendizaje de la Programación en Ingeniería de Sistemas a partir de tres modelos de trabajo en grupo, Entre Ciencia e Ingeniería, vol. 13, no. 26, pp. 43-50, julio-diciembre 2019. DOI: https://doi.org/10.31908/19098367.1158

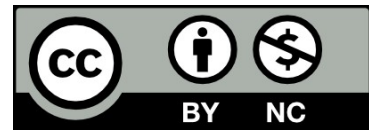

Attribution-NonCommercial 4.0 Intenational (CC By-NC 4.0) course in Systems Engineering. To reach that we worked during 6 semesters and using three models: $4 Q$ model, talent model and random model. We used observation, interaction, and dialogue, qualitative and quantitative results to find the target of this research project. The results show characteristics in the interactions inside the different models of groups. We concluded that it is possible to find an excellence model of teamwork depending on the specific course in computer programming.

Keywords. 4Q model, Computer programming, Learning, Random Model, Systems Engineering, Teamwork.

\section{INTRODUCCIÓN}

$\mathrm{E}^{\mathrm{N}}$ el proceso de formación universitario, una de las competencias más complejas que difícilmente se logran es el desarrollo de habilidades para formar parte de grupos de trabajo (teamworks) y alcanzar objetivos conjuntos a partir de la complementación de sus talentos y capacidades. Tal es el caso de los Ingenieros de Sistemas que, a nivel profesional, deben formar parte frecuentemente de grupos de trabajo conformados por profesionales de diferentes áreas (contadores, economistas, administradores, ingenieros de otras áreas, matemáticos, etc.).

Una de las quejas más sentidas de los empresarios radica en que, a pesar de su alta capacidad técnica, tecnológica y metodológica, la participación de los recién egresados en grupos de trabajo es notoriamente deficiente dado que la dinámica propia de estos grupos invita a pensar en actividades como debates, argumentaciones, foros, interacciones, coincidencias y discrepancias. A la luz de estas necesidades, los docentes de Ingeniería de Sistemas tienen el reto de formar a los estudiantes en estas lides de manera que su salto a la vida profesional no solo consista en participar de las soluciones que, desde su conocimiento científico, la sociedad le exige sino también en convertirse en un ser social que interactúe y participe de las dinámicas de los grupos de trabajo en el ámbito empresarial.

¿Cuál ha de ser el modelo apropiado para la conformación de grupos, en el ámbito académico, de manera que el estudiante pueda desarrollar la competencia de formar parte 
activa, efectiva, eficiente y eficaz de un grupo o equipo de trabajo? Esa podría ser la pregunta de investigación que se trata de responder con el contenido de este artículo y para la cual se aportan elementos de discusión bien para que se adopten en el aula de clases en los procesos de formación en ingeniería o bien para que se tengan nuevos elementos de juicio para nutrir la discusión al respecto.

En especial, en los programas de Ingeniería, a pesar de que como estrategia de trabajo académico se acude a la conformación de grupos de trabajo, pocas veces se analiza la incorporación de esta estrategia desde la óptica de modelos científicos definidos y estructurados que permitan inferir conclusiones con impacto en lo pedagógico. Lo que podría considerarse novedoso en este artículo es que propende por establecer criterios de conformación basados en modelos claramente definidos y que, además, proporciona elementos de juicio para establecer parámetros comparativos en relación con la interacción de los estudiantes y sus respectivos grupos de trabajo.

Esta investigación se justifica pues con frecuencia en los programas de ingeniería, se hace necesario que se desarrollen tareas a partir de lo que hagan los estudiantes en grupo. Si bien la conformación aleatoria de grupos es posiblemente la técnica más utilizada en los programas de formación superior debe aceptarse que no siempre es la más acertada debido a que el estudiante, especialmente el de primeros semestres, queda a discreción de lo que la suerte le depare en la caracterización de sus compañeros referente a sus talentos y capacidades.

Por lo dicho, el objetivo de este artículo es presentar los resultados obtenidos del desarrollo del proyecto asociado dentro del marco de una investigación que teniendo elementos cuantitativos fundamenta sus inferencias a partir de los criterios cualitativos que la componen y que incluyen estudios de casos individuales y grupales con el objetivo de mejorar la conformación de "teamworks" en el aula.

¿Es posible fortalecer el trabajo en grupo, capitalizar sus bondades para posibilitar el logro de objetivos individuales de aprendizaje de los estudiantes de programación de computadores en Ingeniería de Sistemas y, además, de propiciar las actividades propias de la dinámica de los grupos como un camino para fomentar la participación profesional de los futuros ingenieros en grupos y equipos de trabajo? La respuesta, en parte, es el contenido de este artículo que, desde la experiencia, los resultados y las conclusiones aporta elementos de juicio para la discusión al respecto.

El artículo se presenta bajo el esquema del formato internacional IMRYD según el cual se hace una introducción, posteriormente se presentan unos elementos que se destacan dentro del marco teórico correspondiente, se plantea la metodología que se utilizó y luego se presentan los resultados obtenidos a la luz de dicha metodología. Finalmente se hace una discusión al respecto de los resultados obtenidos de la cual se infieren algunas conclusiones terminando con las referencias bibliográficas que se utilizaron para su desarrollo.

\section{MARCO TEÓRICO}

El aprendizaje, a través de procesos en el aula y fuera de ella, puede definirse como el acto a través del cual se adquiere, se modifica o se refuerza el conocimiento existente, los comportamientos, las habilidades, los valores o las preferencias de manera que pueden conducir a un cambio en la sintetización de la información, a una profundización del mismo conocimiento, a una actitud o comportamiento relativos a un tipo y rango de experiencias [1] razón por la cual puede enfatizarse en que es importante que los educadores tengan conocimiento sobre el funcionamiento del cerebro para tener información adicional sobre el proceso de aprendizaje [2].

La habilidad de aprender es una capacidad que tienen los humanos, los animales, las plantas y algunas máquinas. El progreso a través del tiempo tiende a seguir una determinada curva de aprendizaje. El aprendizaje no sucede de una vez, se construye y adquiere forma a partir del conocimiento previo [3]. En este sentido, el aprendizaje puede ser visto como un proceso, más que una colección de hechos y procedimientos, que produce cambios en los organismos y los cambios producidos son, de alguna manera, permanentes. El aprendizaje humano ocurre como parte de los procesos educativos, el desarrollo personal, la incorporación a la escuela o el entrenamiento y el aprendizaje colaborativo brinda posibilidades interesantes en el proceso educativo, dentro del cual el estudiante interactúa permanentemente en el ambiente formal e informal donde se moviliza y en los que aprende, trascendiendo lo institucional [4].

Dicho aprendizaje está orientado por determinados objetivos y se soporta en la motivación [5] y de acuerdo con el área de estudio y el grado de interés, puede desarrollar unas preferencias o modos de aprender más que otros, y esto sumado a unas determinadas maneras de aprender constituyen su estilo de aprendizaje [6]. El estudio de la forma como sucede el aprendizaje es parte de la sicología educativa, la neurosicología, la teoría del aprendizaje y la pedagogía. El aprendizaje puede ocurrir como resultado de los condicionamientos clásicos o habituales, que pueden ser observados en muchas especies animales o como el resultado de actividades más complejas como los juegos, lo cual puede ser observado solo en animales inteligentes [7] de allí el por qué la verdadera situación dentro del aula poco cambia pues muchas de estas ideas se quedan para los congresos, y al volver al aula el docente sigue actuando tradicionalmente y sin innovaciones [8].

El aprendizaje puede ocurrir consciente o inconscientemente. Existe evidencia de que el comportamiento humano se afecta por el aprendizaje prenatal lo cual ha sido observado a lo largo de las 32 semanas de gestación, indicando que el sistema nervioso central está suficientemente desarrollado y motivado por el aprendizaje y la memoria en estas etapas tempranas de la formación. El juego ha sido aprovechado por diferentes teóricos como la primera forma de aprendizaje. Los niños que experimentan el mundo aprenden las reglas y aprenden a interactuar jugando [9]. Algunos científicos piensan que el juego es esencial para el desarrollo de los niños, debido a que les permite encontrar significado a su entorno, a sus interacciones y a su relación con ellos. Según lo anterior la enseñanza se dispone como un proceso en el que intervienen agentes como docente (quien enseña), estudiante (quien aprende), contexto y currículum; y a su vez, la enseñanza depende directamente del término "aprendizaje" [10]. 
En el mundo moderno, la labor del docente universitario va mucho más allá de simplemente impartir un conocimiento desde lo puramente magistral [11]. Existe una gran cantidad de estrategias que posibilitan que un estudiante pueda recorrer un camino más expedito para adquirir conocimiento y estas estrategias incluyen, entre muchas otras, el trabajo en grupo en donde el factor humano y de interacción es considerado de gran importancia en el proceso de aprendizaje [12]. Unirse con otras personas para alcanzar un objetivo de aprendizaje representa cinco ventajas que, en tiempos modernos, son de gran importancia ya que éstas moldean sus competencias de forma que pueda articularse con las dinámicas laborales [13].

El trabajo en grupo posibilita a) interactuar con otras personas y establecer cercanías, b) confrontar ideas y argumentarlas, c) escuchar ideas diferentes con pensamiento crítico, d) unir esfuerzos, habilidades, destrezas y competencias con otros en bien de un objetivo y e) aprender colectivamente. Los grupos de trabajo constituyen, sin lugar a duda, una de las estrategias más comúnmente adoptadas por los docentes universitarios para el desarrollo de sus actividades pedagógicas de cara al logro de sus objetivos [14].

La revisión del término "teamwork" (grupo de trabajo) se utiliza como un mecanismo para referirse a una serie de procesos comportamentales y como un estado emergente que surge cuando un grupo de personas quieren alcanzar un objetivo que le es común. En el mundo de la medicina, el concepto de "teamwork" es un proceso dinámico que involucra dos o más profesionales con habilidades y conocimientos complementarios, que comparten objetivos comunes y que se han ejercitado en esfuerzos conjuntos tanto físicos como mentales para asesorar, planear o evaluar los cuidados de un paciente.

En el mundo de los negocios, las técnicas conjuntas de acompañamiento pueden ser usadas para proveer medidas financieras que resultan más rentables [15]. Las personas que desarrollan políticas de salud, por ejemplo, cada vez acuden más a los grupos de trabajo como un camino para asegurar la calidad y la seguridad en la prestación de sus servicios. En Ingeniería, el trabajo en grupo se hace altamente necesario toda vez que el ingeniero tendrá que interactuar siempre con profesionales de otras áreas permanentemente.

El ingeniero de sistemas es posiblemente el profesional de la ingeniería que más requiere de esa interacción con otros profesionales dado que si su objetivo profesional gira alrededor de la información, son otros profesionales los que pueden ilustrarle acerca del uso de ésta [16]. La relación que se establece a nivel empresarial cuando un ingeniero de sistemas interactúa con contadores, economistas, ingenieros de otras áreas, administradores, etc. le provee miradas que enriquecen su propio perfil y favorece las razones que justifican su presencia en una empresa [17].

El primer modelo que se ha adoptado para esta investigación se conoce como modelo $4 \mathrm{Q}$ de preferencias de pensamiento y corresponde a un modelo diseñado para ayudar a los investigadores, a los equipos, a los grupos de trabajo y a las empresas a alcanzar los mejores beneficios de las personas que dirigen. Este modelo reconoce que mientras diferentes tareas requieren procesos mentales disímiles, y las personas tienen formas de pensar distintas, las organizaciones pueden obtener unos resultados mejores si conocen el espectro posible de pensamientos y enfoques que tienen los seres humanos [18].

Los seres humanos tienen preferencias de pensamiento que, en algunos son muy fuertes y en otros son más moderadas. Dos personas podrían tener puntos de vista distintos y coincidir en sus preferencias de pensamiento [19]. El modelo de preferencias de pensamiento llamado 4Q indica que cada uno tiene acceso a cuatro formas de pensamiento y que una de ellas domina a los demás cuadrantes. La Fig. 1 presenta un diagrama del modelo $4 \mathrm{Q}$ referenciado.

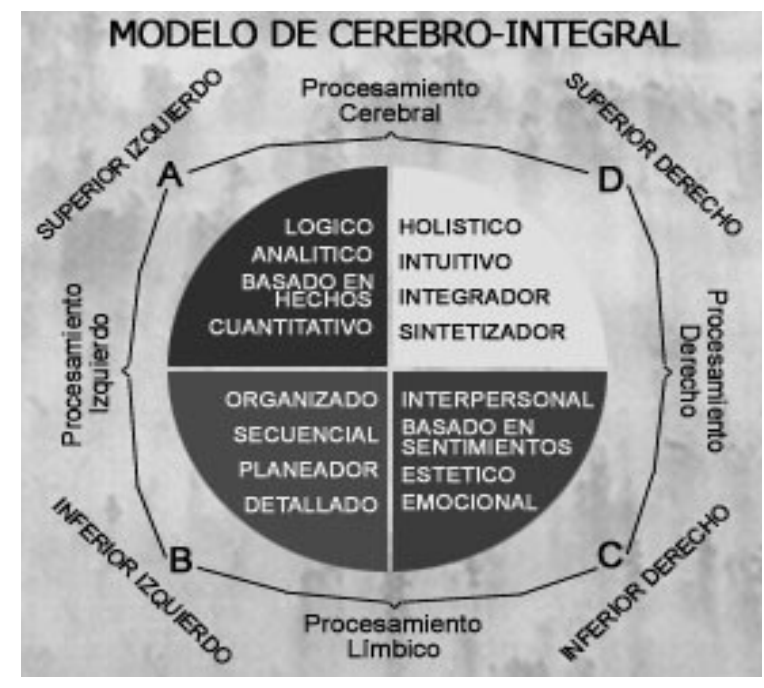

Fig. 1, MODELO 4Q DE PREFERENCIAS DE PENSAMIENTO HERRMANN http://herrmannlatin.com/hbdi.php

De acuerdo con este modelo, el cuadrante A corresponde al pensamiento lógico analítico que es el que permite que las personas con este pensamiento preferente sean las más indicadas para recoger datos, realizarles un análisis, comprender cómo trabajan las cosas, evaluar ideas basadas en hechos y también para el razonamiento lógico. En el cuadrante B se ubica el pensamiento secuencial y con él se ubican aquellos en quienes éste es su cuadrante preferente que normalmente son muy hábiles para seguir linealmente un conjunto de instrucciones, para realizar trabajos claramente orientados, para encontrar solución a un problema desarrollándola paso a paso, así como para organizar e implementar una determinada solución.

El cuadrante $\mathrm{C}$ tiene relación con el pensamiento interpersonal, es el cuadrante social según el cual las personas desarrollan una mayor capacidad tanto para escuchar como para expresar sus ideas, para buscar y encontrar aquello que pareciera importante para las demás, para analizar las características de grupos de interacción y, en algunos casos, para liderarlos. El $4^{\circ}$ cuadrante es el cuadrante D o imaginativo. En este cuadrante se pueden ubicar aquellas personas que son capaces de observar el paisaje y que no se detienen a mirar solamente un árbol, que pueden tomar la iniciativa y que asumen retos por ellos mismos. Son mucho más visuales y tienen una forma de pensar con un alto componente metafórico lo que les permite poseer una capacidad de resolver creativamente los problemas además de tener un pensamiento que se distingue a largo plazo por sus características. 
El segundo modelo que se ha adoptado en esta investigación se llama Modelo de talento académico según el cual la clasificación de estudiantes se basa en la experiencia tanto del autor de este artículo como de otros docentes y/o investigadores. Los estudiantes pueden ubicarse en una de tres categorías: estudiantes Altamente Talentosos (AT), estudiantes Medianamente Talentosos (MT) y estudiantes Bajamente Talentosos (BT).

Los estudiantes Altamente Talentosos (AT) se pueden definir como los que ya tienen un conocimiento disciplinar sólido, que tanto sus habilidades como sus competencias están en rima con dicho conocimiento disciplinar y que saben aprovecharlo. Por su parte, los estudiantes Bajamente Talentosos (BT) son aquellos que cumplen una de las siguientes tres condiciones: a) no poseen un conocimiento disciplinar en ningún nivel, b) tanto sus habilidades como sus competencias parecieran no estar en rima con lo que la asignatura $\mathrm{y} / \mathrm{o}$ el programa de formación profesional requiere y c) normalmente no manifiestan un interés pronunciado para involucrarse en aquellas situaciones donde dicho conocimiento se puede aplicar. Los estudiantes Medianamente Talentosos (MT) pueden definirse como aquellos que aunque tienen un conocimiento disciplinar débil, parecen tener habilidades y competencias que coinciden con los requerimientos del programa de formación pero que aún no ha aflorado en ellos y por ende ni siquiera ellos, muchas veces, lo saben; además participan tímidamente en las situaciones en las cuales se requiere aplicar el conocimiento aunque muestran gran interés en estar allí.

El tercer modelo que se ha adoptado en esta investigación corresponde al modelo aleatorio de agrupamiento según el cual el estudiante hace grupo con cualquier persona sin que medie ningún modelo específico. Es de anotar que este modelo de agrupamiento es completamente aleatorio cuando se incorpora como estrategia en las asignaturas de los primeros semestres $\mathrm{y}$, de manera muy especial, en el $1^{\mathrm{o}}$ semestre de cualquier programa de formación profesional. Este es el modelo al cual se enfrenta el ingeniero de sistemas en su vida profesional salvo aquellos casos en los cuales tiene la potestad de escoger su equipo de trabajo. Es allí donde su capacidad de creatividad y manipulación de los lenguajes de programación se pone a prueba [20].

La enseñanza y el aprendizaje de la programación de computadores se ha convertido cada vez en una necesidad más alta que establece un reto en dos sentidos: al estudiante le impone el reto de aprender a programar, es decir, de apropiar, asimilar, aplicar y evaluar las técnicas, teorías, tecnologías, modelos y metodologías que subyacen a un determinado paradigma de programación [21]. Por su parte el docente tiene el reto de adoptar estrategias que le posibiliten al estudiante alcanzar sus logros de aprendizaje [22], es decir, aprender a programar, por caminos que fomenten la apropiación del conocimiento disciplinar y que, además, lo formen para incorporarse a la vida profesional de la manera más efectiva [23].

\section{METODOLOGÍA}

Este estudio se realizó con estudiantes de la asignatura Programación I del $1^{\circ}$ semestre de Ingeniería de Sistemas y Computación de la Universidad Tecnológica de Pereira durante los semestres I 2015, II 2015, I 2016, II 2016, I 2017 y II 2017. Cada estrategia de conformación de grupos según el modelo adoptado (modelo 4Q, modelo de rendimiento académico, modelo aleatorio) se aplicó con un grupo diferente. El contenido de la asignatura se dividió en 4 módulos cada uno con duración de 4 semanas y temáticas específicas tratadas en cada módulo. La tabla I presenta la organización de la asignatura.

TABLA I

\begin{tabular}{|c|c|c|}
\hline & & \\
\hline Módulo & Semana & Contenido \\
\hline \multirow{4}{*}{$\begin{array}{c}\text { Módulo } \\
1\end{array}$} & Semana 1 & ¿Cómo se programa? \\
\hline & Semana 2 & Funciones simples \\
\hline & Semana 3 & Enlace de funciones \\
\hline & Semana 4 & Ejercicios \\
\hline \multirow{4}{*}{$\begin{array}{c}\text { Módulo } \\
2\end{array}$} & Semana 5 & Condicionales simples \\
\hline & Semana 6 & Condicionales múltiples \\
\hline & Semana 7 & Menús \\
\hline & Semana 8 & Ejercicios \\
\hline \multirow{4}{*}{$\begin{array}{c}\text { Módulo } \\
3\end{array}$} & Semana 9 & Recursividad Nivel I \\
\hline & Semana 10 & Recursividad Nivel II \\
\hline & Semana 11 & Recursividad Nivel III \\
\hline & Semana 12 & Ejercicios \\
\hline \multirow{4}{*}{$\begin{array}{c}\text { Módulo } \\
4\end{array}$} & Semana 13 & Listas \\
\hline & Semana 14 & Vectores \\
\hline & Semana 15 & Desarrollo de Aplicaciones \\
\hline & Semana 16 & Ejercicios \\
\hline
\end{tabular}

En cada semestre se trabajaron con tres semestres de manera simultánea. Con uno de ellos se adoptó la estrategia de conformación de grupos de trabajo basándose en el modelo $4 \mathrm{Q}$, con otro se adoptó el modelo de conformación por rendimiento académico y con el otro se adoptó la conformación de aleatoria de grupos. A través de observación directa, pruebas escritas, modelos de caracterización de estudiantes y entrevistas personalizadas se logró ubicar a los estudiantes en cada una de las categorías de cada modelo. Algunas caracterizaciones fueron un poco más dispendiosas por su naturaleza científica tal como el modelo 4Q. El modelo por rendimiento académico se basó en información cuantitativa de cada estudiante involucrado según sus informes de notas del colegio. El modelo de conformación aleatoria no tuvo ninguna dificultad por su misma naturaleza.

En cuanto a la conformación de los grupos de trabajo bajo el modelo 4Q, éstos se conformaron de manera que siempre los grupos tuvieran representantes de los 4 cuadrantes. Lo mismo se mantuvo con los grupos conformados por rendimiento académico en los grupos en donde se adoptaron. La conformación aleatoria solo tuvo como referente, la cantidad promedio de grupos conformados bajo los modelos $4 Q$ y de rendimiento académico.

Siempre se mantuvo la organización de estos grupos durante el semestre. A todos los estudiantes se les impartieron las mismas clases magistrales, las mismas explicaciones, se les colocaron los mismos talleres y los mismos parciales para que fueran resueltos en grupo. De eso precisamente es que se trataba esta parte del proyecto de investigación. La asignatura contó con 4 evaluaciones parciales con un peso porcentual cada una del $15 \%$. El examen final escrito tenía un peso porcentual de $30 \%$ y se concedió un $10 \%$ de motivación equivalente a una nota 5.0 para todos los estudiantes. A lo largo de cada semestre se presentaron algunas deserciones que 
obedecieron a razones ajenas al proceso de investigación como reubicación en otra ciudad, transferencia de universidad, transferencia de programa, situaciones familiares apremiantes y cambios en la situación económica.

\section{RESULTADOS}

La tabla II muestra la cantidad de estudiantes que participaron en este estudio, discriminados por grupo.

TABLA II

ESTUDIANTES INVOLUCRADOS EN EL ESTUDIO
\begin{tabular}{|c|l|c|l|c|c|}
\hline \multirow{2}{*}{ Año } & \multicolumn{2}{|c|}{ Semestre I } & \multicolumn{2}{c|}{ Semestre II } & \multirow{2}{*}{ Tot } \\
\cline { 2 - 6 } & Grp & Est & Grp & Est & \\
\hline \multirow{3}{*}{2015} & $4 Q$ & 21 & $4 Q$ & 20 & 41 \\
\cline { 2 - 6 } & RA & 19 & RA & 23 & 42 \\
\cline { 2 - 6 } & Aleat & 20 & Aleat & 21 & 41 \\
\hline \multirow{3}{*}{2016} & $4 Q$ & 23 & $4 Q$ & 23 & 46 \\
\cline { 2 - 6 } & RA & 20 & RA & 19 & 39 \\
\cline { 2 - 6 } & Aleat & 19 & Aleat & 18 & 37 \\
\hline \multirow{3}{*}{2017} & $4 Q$ & 20 & $4 Q$ & 21 & 41 \\
\cline { 2 - 6 } & RA & 21 & RA & 20 & 41 \\
\cline { 2 - 6 } & Aleat & 22 & Aleat & 21 & 43 \\
\hline To o a 1 & 185 & & 186 & 371 \\
\hline
\end{tabular}

En total, 371 estudiantes participaron en el estudio. En cada semestre se designó un grupo completo para trabajar con la conformación de grupos basado en el modelo 4Q (4Q), otro grupo completo en donde los grupos se conformaron basados en su rendimiento académico (RA) y un tercer grupo en donde se permitió que los estudiantes se organizaran aleatoriamente (Aleat). La tabla III presenta la cantidad de "teamworks" que se conformaron en cada grupo.

Se procuró que los teamworks de los grupos basados en el modelo 4Q tuvieran al menos 1 exponente de cada categoría del modelo (Lógico, Secuencial, Imaginativo y Social). De la misma forma se procuró, al máximo, que los teamworks de los grupos basados en el modelo de rendimiento académico contaran con la participación de, por lo menos, 2 exponentes de cada categoría (Altamente Talentosos, Medianamente Talentosos y Bajamente Talentosos). La conformación aleatoria de teamworks no tuvo ningún tipi de indicación por lo tanto se procuró que se conformara una cantidad equivalente a los de los grupos en donde se aplicaban las otras metodologías.

TABLA III

TEAMWORKS CONFORMADOS

\begin{tabular}{|c|l|c|l|c|c|}
\hline \multirow{2}{*}{ Año } & \multicolumn{2}{|c|}{ Semestre I } & \multicolumn{2}{|c|}{ Semestre II } & \multirow{2}{*}{ Tot } \\
\cline { 2 - 6 } & Grp & Est & Grp & Est & \\
\hline \multirow{3}{*}{2015} & $4 Q$ & 4 & $4 Q$ & 4 & 8 \\
\cline { 2 - 6 } & RA & 6 & RA & 7 & 13 \\
\cline { 2 - 6 } & Aleat & 7 & Aleat & 6 & 13 \\
\hline \multirow{3}{*}{2016} & $4 Q$ & 5 & $4 Q$ & 4 & 9 \\
\cline { 2 - 6 } & RA & 6 & RA & 5 & 11 \\
\cline { 2 - 6 } & Aleat & 4 & Aleat & 4 & 8 \\
\hline \multirow{3}{*}{2017} & 4Q & 5 & $4 Q$ & 4 & 9 \\
\cline { 2 - 6 } & RA & 5 & RA & 6 & 11 \\
\cline { 2 - 6 } & Aleat & 6 & Aleat & 6 & 12 \\
\hline \multicolumn{2}{|c|}{ To t a 1 } & 48 & & 46 & 94 \\
\hline
\end{tabular}

En lo cuantitativo, se presenta en la tabla IV el promedio de las notas parciales de cada curso y de cada grupo obtenidas a lo largo del semestre.
TABLA IV

PROMEDIO DE NOTAS OBTENIDAS

\begin{tabular}{|c|c|c|c|c|c|c|c|}
\hline \multirow{2}{*}{ Año } & \multirow{2}{*}{ Sem } & \multicolumn{2}{|c|}{ 4Q } & \multicolumn{2}{c|}{ R.A. } & \multicolumn{2}{c|}{ Aleat } \\
\cline { 2 - 8 } & Grp1 & Grp2 & Grp1 & Grp2 & Grp1 & Grp2 \\
\hline \multirow{2}{*}{2015} & I & 4,0 & 4,1 & 4,4 & 4,5 & 4,2 & 4,1 \\
\cline { 2 - 8 } & II & 3,6 & 3,8 & 3,9 & 3,9 & 4,0 & 4,1 \\
\hline \multirow{2}{*}{2016} & I & 3,7 & 3,8 & 3,5 & 3,4 & 4,5 & 4,6 \\
\cline { 2 - 8 } & II & 4,1 & 4,3 & 4,3 & 4,1 & 4,2 & 4,2 \\
\hline \multirow{2}{*}{2017} & I & 4,3 & 4,5 & 4,1 & 4,0 & 3,9 & 3,7 \\
\cline { 2 - 7 } & II & 4,0 & 4,1 & 4,0 & 4,1 & 4,4 & 4,4 \\
\hline \multicolumn{3}{|c|}{ Promedio } & \multicolumn{6}{|c|}{4,0} & \multicolumn{3}{c}{4,0} & \multicolumn{2}{c}{4,2} \\
\hline
\end{tabular}

Finalizando el semestre se solicitó que los estudiantes respondieran por escrito dos preguntas. La primera era abierta y cuestionaba su satisfacción en el grupo donde le había correspondido de manera que el estudiante pudiera expresarse libre y anónimamente indicando solamente si había estado en grupo $4 \mathrm{Q}$, grupo RA o grupo aleatorio. La segunda pregunta era cerrada: " ¿A través de la metodología adoptada, aprendió a programar? (explique el porqué)". La tabla VA presenta los resultados obtenidos teniendo en cuenta los siguientes criterios:

- Respuestas Muy Favorables (RMF) aquellas que se concentraron más en las ventajas y cuya respuesta a la segunda pregunta fue afirmativa

- Respuestas Favorables (RF) aquellas que destacaron las ventajas, pero cuya respuesta a la segunda pregunta fue negativa

- Respuestas Desfavorables (RDF) aquellas que destacaron más las desventajas y su respuesta a la segunda pregunta fue positiva

- Respuestas Muy Desfavorables (RMDF) aquellas que destacaron más las desventajas y su respuesta a la segunda pregunta fue negativa.

TABLA V A

TABULACIÓN DE RESPUESTA $1^{\text {a }}$ PREGUNTA

\begin{tabular}{|c|c|c|c|c|c|c|c|}
\hline Año & Sem & Tipo & RMF & RF & RDF & RMDF & Tot \\
\hline \multirow{6}{*}{2015} & \multirow{3}{*}{ I } & $4 Q$ & 16 & 3 & 2 & 0 & \multirow{3}{*}{60} \\
\hline & & R.A. & 15 & 3 & 1 & 0 & \\
\hline & & Aleat & 15 & 3 & 1 & 1 & \\
\hline & \multirow{3}{*}{ II } & $4 Q$ & 14 & 3 & 2 & 1 & \multirow{3}{*}{64} \\
\hline & & R.A. & 17 & 2 & 2 & 2 & \\
\hline & & Aleat & 15 & 4 & 2 & 0 & \\
\hline \multirow{6}{*}{2016} & \multirow{3}{*}{ I } & $4 \mathrm{Q}$ & 19 & 3 & 1 & 0 & \multirow{3}{*}{62} \\
\hline & & R.A. & 14 & 3 & 2 & 1 & \\
\hline & & Aleat & 16 & 2 & 1 & 0 & \\
\hline & \multirow{3}{*}{ II } & $4 Q$ & 18 & 3 & 2 & 0 & \multirow{3}{*}{60} \\
\hline & & R.A. & 15 & 2 & 1 & 1 & \\
\hline & & Aleat & 16 & 1 & 1 & 0 & \\
\hline \multirow{6}{*}{2017} & \multirow{3}{*}{ I } & $4 Q$ & 18 & 1 & 1 & 0 & \multirow{3}{*}{63} \\
\hline & & R.A. & 18 & 1 & 1 & 1 & \\
\hline & & Aleat & 19 & 1 & 1 & 1 & \\
\hline & \multirow{3}{*}{ II } & $4 \mathrm{Q}$ & 15 & 3 & 2 & 1 & \multirow{3}{*}{62} \\
\hline & & R.A. & 16 & 2 & 2 & 0 & \\
\hline & & Aleat & 16 & 3 & 1 & 1 & \\
\hline \multicolumn{3}{|c|}{ Total } & 292 & 43 & 26 & 10 & 371 \\
\hline \multicolumn{3}{|c|}{$\%$} & 78,7 & 11,6 & 7,0 & 2,7 & 100 \\
\hline
\end{tabular}

En relación con la segunda pregunta, la tabla V B presenta los resultados obtenidos según la breve encuesta que se hizo al finalizar el semestre a cada uno de los estudiantes. 
TABLA V B

TABULACIÓN DE RESPUESTAS $2^{\mathrm{a}}$ PREGUNTA

\begin{tabular}{|c|c|c|c|c|c|c|}
\hline Año & Sem & Tipo & $\mathbf{S i}$ & No & $\begin{array}{c}\text { No } \\
\text { Sabe }\end{array}$ & Tot \\
\hline \multirow{6}{*}{2015} & \multirow{3}{*}{ I } & $4 Q$ & 18 & 3 & 0 & \multirow{3}{*}{60} \\
\hline & & R.A. & 17 & 2 & 0 & \\
\hline & & Aleat & 18 & 2 & 0 & \\
\hline & \multirow{3}{*}{ II } & $4 Q$ & 18 & 1 & 1 & \multirow{3}{*}{64} \\
\hline & & R.A. & 20 & 2 & 1 & \\
\hline & & Aleat & 18 & 3 & 0 & \\
\hline \multirow{6}{*}{2016} & \multirow{3}{*}{ I } & $4 Q$ & 20 & 3 & 0 & \multirow{3}{*}{62} \\
\hline & & R.A. & 18 & 1 & 1 & \\
\hline & & Aleat & 16 & 2 & 1 & \\
\hline & \multirow{3}{*}{ II } & $4 Q$ & 20 & 3 & 0 & \multirow{3}{*}{60} \\
\hline & & R.A. & 17 & 2 & 0 & \\
\hline & & Aleat & 16 & 1 & 1 & \\
\hline \multirow{6}{*}{2017} & \multirow{3}{*}{ I } & $4 Q$ & 20 & 0 & 0 & \multirow{3}{*}{63} \\
\hline & & R.A. & 18 & 2 & 0 & \\
\hline & & Aleat & 20 & 2 & 0 & \\
\hline & \multirow{3}{*}{ II } & $4 Q$ & 18 & 2 & 1 & \multirow{3}{*}{62} \\
\hline & & R.A. & 18 & 1 & 1 & \\
\hline & & Aleat & 17 & 3 & 2 & \\
\hline \multicolumn{3}{|c|}{ Tota } & 327 & 35 & 9 & 371 \\
\hline \multicolumn{3}{|c|}{$\%$} & 88,1 & 9,4 & 2,4 & 100 \\
\hline
\end{tabular}

Se adoptó una respuesta "No Sabe" para aquellas respuestas que de manera clara y contundente no son un "Si" ni un "No" absoluto.

\section{DISCUSIÓN}

Se podría pensar que este proceso de investigación se realizara en un curso de II semestre sin embargo esto podría malograr la conformación aleatoria de "teamworks" debido a que los estudiantes ya se conocen y por tanto la conformación libre de grupos de trabajo no sería tan aleatoria como se desea. Tanto el modelo 4Q como el modelo de rendimiento académico y el modelo aleatorio son tres modelos que permiten obtener resultados significativos en el marco de este tipo de investigaciones en el aula. El modelo 4Q tiene todo el respaldo científico razón por la cual para la clasificación de los alumnos se acudió a un instrumento especializado basado en los fundamentos teóricos del modelo.

El modelo de rendimiento académico es un modelo derivado de la experiencia de varios docentes investigadores que permite categorizar a los estudiantes en tres niveles fácilmente detectables a partir de sus notas de secundaria. La conformación de grupos de manera aleatoria es la estrategia que normalmente se utiliza en los salones de clase por parte de los docentes de diferentes programas universitarios. Si bien, es el menos científico de todas formas se adoptó precisamente por ser el más utilizado tanto en la vida académica como en la vida profesional.

Durante el proceso de investigación, y mientras los estudiantes realizaban sus talleres o sus pruebas escritas en grupo, se realizaron las siguientes observaciones:

- En los grupos 4Q normalmente quien tomaba el liderazgo era un estudiante con perfil dominante social (Cuadrante C) y, en algunas ocasiones, se notaba discusión sana y cordiales discrepancias con algún estudiante de cuadrante A (Lógico). La participación del estudiante B (Secuencial) era mucho más operativa y el estudiante de cuadrante dominante D (Imaginativo) tenía la característica de participar mucho en la estructuración de las soluciones haciendo propuestas $\mathrm{y}$, algunas veces, yendo mucho más allá de lo escrito, aunque su participación siempre fue baja. La solución a los problemas y enunciados planteados venía de la mano del estudiante con cuadrante dominante Lógico

- En los grupos de talento académico, se notó que el liderazgo siempre lo tomaba el estudiante AT (Altamente Talentoso) aunque en algunas oportunidades se notaba que se daba a la tarea de explicar sus soluciones, aunque pocas veces posibilitaba espacios para el debate y la discusión. Los estudiantes BT (Bajamente Talentosos) poco participaban en el proceso, aunque mostraban alta voluntad de realizar las tareas operativas. Los estudiantes MT (Medianamente Talentosos) intentaban siempre participar en la estructuración de las soluciones, trataban de discutir lo que correspondiera y generalmente adoptaban la tarea de ser los auditores del trabajo del estudiante BT indicándole lo que debía hacer y cómo debía hacerlo, en consonancia con las indicaciones del estudiante AT. Normalmente la solución a los problemas surgió por iniciativa del estudiante tipo AT

- En los grupos aleatorios siempre se notaba que existían por lo menos dos líderes y que los debates y discusiones se daban entre ellos mientras los demás esperaban pacientemente para que les indicaran qué debían hacer. Fue el tipo de grupo en el cual se realizaron mayores debates y discusiones. Las soluciones terminaban siendo concertadas a partir de dichos debates. Al final todos participaban activamente.

La tabla VI presenta un resumen de las características detectadas al interior de cada grupo a partir de su dinámica de interacción y tomando como base la observación del docente investigador.

TABLA VI

TABLA DE ANÁLISIS DE LOS GRUPOS

\begin{tabular}{|c|c|c|}
\hline $\begin{array}{l}\text { Team } \\
\text { work }\end{array}$ & Tópico & Análisis \\
\hline \multirow{8}{*}{$\begin{array}{c}\text { Mod. } \\
\text { 4Q }\end{array}$} & Confiabilidad & $\begin{array}{l}\text { Se requiere ubicar a cada estudiante en su } \\
\text { cuadrante preferente a partir de la } \\
\text { observación y el diálogo con cada uno lo cual } \\
\text { implica mucho tiempo e instrumentos. } \\
\text { Científicamente el modelo es totalmente } \\
\text { confiable }\end{array}$ \\
\hline & Participación & $\begin{array}{l}\text { Los estudiantes participan en proporciones } \\
\text { diferentes, pero todos se hacen oír }\end{array}$ \\
\hline & $\begin{array}{l}\text { Papel de cada } \\
\text { estudiante }\end{array}$ & $\begin{array}{l}\text { El estudiante Lógico (A) junto con el Social } \\
\text { (C) son los líderes. La parte operativa la } \\
\text { realiza el estudiante Secuencial (B) y el } \\
\text { estudiante imaginativo } \\
\text { solución }\end{array}$ \\
\hline & $\begin{array}{l}\text { Discusiones y } \\
\text { Debates }\end{array}$ & $\begin{array}{l}\text { Las discusiones son breves, no se extienden } \\
\text { mucho y normalmente se le concede la razón } \\
\text { al estudiante Lógico (A) }\end{array}$ \\
\hline & $\begin{array}{l}\text { Planteamiento } \\
\text { de Soluciones }\end{array}$ & $\begin{array}{l}\text { Normalmente se adoptan las soluciones que } \\
\text { plantea el estudiante Lógico (A) dado que sus } \\
\text { argumentos son más claros }\end{array}$ \\
\hline & $\begin{array}{l}\text { Trabajo } \\
\text { Operativo }\end{array}$ & $\begin{array}{l}\text { La parte operativa la realiza el estudiante } \\
\text { Secuencial (B), nadie riñe con él por esta } \\
\text { labor. Este estudiante es el que menos } \\
\text { participa en las discusiones }\end{array}$ \\
\hline & Concertación & $\begin{array}{l}\text { La concertación siempre se da alrededor de la } \\
\text { solución que propone el estudiante Lógico } \\
\text { (A) }\end{array}$ \\
\hline & Interacción & $\begin{array}{l}\text { Fuera del grupo de trabajo interactúan muy } \\
\text { poco entre sí, se limitan a conversar durante } \\
\text { la sesión de trabajo }\end{array}$ \\
\hline $\begin{array}{l}\text { Mod. } \\
\text { Rend. } \\
\text { Acad. }\end{array}$ & Confiabilidad & $\begin{array}{l}\text { El modelo es bastante claro y es fácil de } \\
\text { detectar a los estudiantes para ubicarlos en } \\
\text { cada categoría }\end{array}$ \\
\hline
\end{tabular}




\begin{tabular}{|c|c|c|}
\hline & Participación & $\begin{array}{l}\text { Los estudiantes participan de manera activa, } \\
\text { pero se nota más liderazgo del estudiante AT. } \\
\text { Los demás aceptan sus propuestas de solución }\end{array}$ \\
\hline & $\begin{array}{l}\text { Papel de cada } \\
\text { estudiante }\end{array}$ & $\begin{array}{l}\text { El estudiante AT propone soluciones, el } \\
\text { estudiante BT realiza la parte puramente } \\
\text { operativa y el estudiante MT, a partir de } \\
\text { dudas y preguntas, dinamiza la solución que } \\
\text { se adopta finalmente }\end{array}$ \\
\hline & $\begin{array}{l}\text { Discusiones y } \\
\text { Debates }\end{array}$ & $\begin{array}{l}\text { Hay muy poca discusión, los estudiantes MT } \\
\text { y BT se ajustan a lo que propone el estudiante } \\
\text { AT. Hay bastantes preguntas por parte del } \\
\text { estudiante MT }\end{array}$ \\
\hline & $\begin{array}{l}\text { Planteamiento } \\
\text { de Soluciones }\end{array}$ & $\begin{array}{l}\text { La solución siempre la plantea el estudiante } \\
\text { AT y los demás miembros del grupo la } \\
\text { aceptan }\end{array}$ \\
\hline & $\begin{array}{l}\text { Trabajo } \\
\text { Operativo }\end{array}$ & $\begin{array}{l}\text { Toda la parte operativa es realizada por el } \\
\text { estudiante BT con la supervisión del } \\
\text { estudiante MT }\end{array}$ \\
\hline & Concertación & $\begin{array}{l}\text { No hay concertación como producto de un } \\
\text { debate, solo aceptan las soluciones que } \\
\text { plantea el estudiante AT }\end{array}$ \\
\hline & Interacción & $\begin{array}{l}\text { Por fuera del grupo, tienen una interacción } \\
\text { moderada }\end{array}$ \\
\hline \multirow{8}{*}{$\begin{array}{l}\text { Mod. } \\
\text { Aleat. }\end{array}$} & Confiabilidad & $\begin{array}{l}\text { El modelo es absolutamente aleatorio, es el } \\
\text { más sencillo pues no requiere ningún tipo de } \\
\text { estudio, preparación o experiencia para } \\
\text { conformar los grupos }\end{array}$ \\
\hline & Participación & $\begin{array}{l}\text { Los estudiantes intentan participar en la } \\
\text { misma proporción, se sienten todos al mismo } \\
\text { nivel, aunque se detecta que hay algunos } \\
\text { estudiantes más talentosos que otros }\end{array}$ \\
\hline & $\begin{array}{l}\text { Papel de cada } \\
\text { estudiante }\end{array}$ & $\begin{array}{l}\text { Se distribuyen tareas y todos intentan } \\
\text { cumplirlas con la mayor voluntad. Entre todos } \\
\text { se dan a la tarea de supervisar lo que hacen } \\
\text { los otros }\end{array}$ \\
\hline & $\begin{array}{l}\text { Discusiones y } \\
\text { Debates }\end{array}$ & $\begin{array}{l}\text { Hay mucha discusión y mucho debate, se } \\
\text { plantean diferentes soluciones, se discuten y } \\
\text { se presentan con buenos argumentos }\end{array}$ \\
\hline & $\begin{array}{l}\text { Planteamiento } \\
\text { de Soluciones }\end{array}$ & $\begin{array}{l}\text { La solución se escoge a partir de la } \\
\text { concertación que se deriva de los argumentos } \\
\text { que cada uno presente }\end{array}$ \\
\hline & $\begin{array}{l}\text { Trabajo } \\
\text { Operativo }\end{array}$ & $\begin{array}{l}\text { El trabajo operativo es asignado también } \\
\text { aleatoriamente. Todo el que recibe esta } \\
\text { designación lo hace de buen agrado }\end{array}$ \\
\hline & Concertación & $\begin{array}{l}\text { Siempre existe concertación en la solución a } \\
\text { adoptar frente a un enunciado o un problema } \\
\text { planteado }\end{array}$ \\
\hline & Interacción & $\begin{array}{l}\text { Por fuera del grupo tienen una alta } \\
\text { interacción, parecieran proyectar la imagen de } \\
\text { ser amigos dentro y fuera del grupo }\end{array}$ \\
\hline
\end{tabular}

La tabla II permite inferir que, de los 371 estudiantes que participaron en el estudio que inspira este artículo, 169 lo hicieron a luz de la conformación de grupos basados en el modelo 4Q, 122 lo hicieron basados en el modelo de rendimiento académico y 121 lo hicieron bajo el modelo aleatorio que corresponden al $46 \%, 33 \%$ y $33 \%$ del total de estudiantes, respectivamente. Es una muestra suficientemente amplia para realizar las inferencias a que haya lugar. El desbalance en las proporciones se origina en la designación de los grupos que, para el estudio, resulta ser de poca importancia.

De acuerdo con los datos de la tabla III, se puede verificar que a la luz del modelo 4Q se conformaron 26 teamworks, con el modelo de rendimiento académico fueron 35 grupos de trabajo y con el modelo aleatorio fueron 33 grupos de trabajo conformados, que corresponden al $28 \%, 37 \%$ y $35 \%$, respectivamente, de los 94 grupos conformados en total durante los 6 semestres que duró el estudio. La proporción de los grupos de trabajo difiere de la proporción de la cantidad de estudiantes debido a que aquellos parten de la aplicación de un modelo específico mientras que éstos parten de la forma como se hayan distribuido los cursos desde la Dirección del programa.

En los datos de la tabla IV se puede observar que los promedios en los grupos de trabajos conformados bajo el modelo 4Q y bajo el modelo de rendimiento académico son iguales $(4,0)$ mientras que el promedio de notas de los grupos de trabajo conformados aleatoriamente tiene un valor más alto $(4,2)$ que, para la investigación y considerando el tamaño y características de la muestra, resulta ser significativo.

Por su parte, tal como se presenta en la tabla V A, las respuestas muy favorables (RMF) corresponden a una proporción del 78,7\% lo cual indica, según los parámetros que se definieron, que los estudiantes participantes se concentraron más en las ventajas del proceso de aprendizaje y su respuesta a la segunda pregunta (que se analiza a continuación) fue afirmativa.

En la tabla VB se observa que a la pregunta "¿A través de la metodología adoptada, aprendió a programa?", la respuesta afirmativa "Si" correspondió a un $88,1 \%$ lo cual valida el proceso en su objetivo fundamental como era el de proveer un camino para que se apropiaran los conceptos básicos de un primer curso de programación en Ingeniería de Sistemas y Computación.

\section{CONCLUSIONES}

De acuerdo con los resultados obtenidos se puede concluir que la adopción de cualquiera de los tres modelos a que se hace referencia en este artículo es una estrategia válida en un proceso de aprendizaje de la programación y que la investigación provee elementos de juicio comparativos que permiten vislumbrar algunas ventajas de cada modelo frente a los otros dos. El promedio de 4,2 en las notas de las evaluaciones parciales y evaluación final del curso permite plantear la conveniencia de que en cursos iniciales de programación de computadores en un programa de Ingeniería de Sistemas, el modelo aleatoria de conformación de grupos resulta ser un modelo muy interesante tanto en lo cuantitativo como en lo cualitativo, tal como se expone en la tabla 4.

Esta estrategia podrá ser, en los primeros semestres, una muy buena aproximación a la vida real en la cual un Ingeniero de Sistemas tendrá que interactuar con grupos interdisciplinarios sobre cuyos miembros no se tiene ninguna injerencia al momento de su selección. Es de anotar que la conformación aleatoria de grupos de trabajo implica, por parte del docente, un acompañamiento en la forma como un "Teamwork" puede trabajar de manera eficiente y un monitoreo permanente para que la formación universitaria surta los efectos que se esperan en la transformación de estudiante a profesional.

\section{REFERENCIAS}

[1] Attard, A., Di Ioio, E., \& Geven, K. "Student Centered Learning. An insight into theory and practice". Bucarest: Lifelong learning programme - European Community. 2010.

[2] Gonzalez, A., \& Palomeque, L. "Integración de estrategias didácticas y neurocientíficas para mejorar la motivación y el aprendizaje en cursos 
de química básica”. Revista Entre Ciencia e Ingeniería, vol. 11, no. 11, pp. 89-94. Junio 2017.

[3] Ausubel, D. Sicología Educativa: Un punto de vista cognoscitivo. Ciudad de México: Trillas. 1986. p. 86.

[4] Montes, J., Escobar, R., \& Cadavid, G. "Uso de herramientas tecnológicas en el desarrollo de un curso de Matemáticas 1 en la Universidad Tecnológica de Pereira”. Revista Entre Ciencia e Ingeniería, vol. 12, no. 23, pp. 66-71. Junio 2018.

[5] Bruner, J. Actos de Significado: Mas allá de la revolución cognitiva. Boston, MA: Alianza Editorial SA. 2009. P. 59.

[6] Yacub, B., Patron, G., Agámez, M., \& Acevedo, D. "Estilos de aprendizaje y su relación con repitencia y retraso académico en Ingeniería Biomédica, Electrónica e Industrial". Revista Entre Ciencia e Ingeniería, vol. 12, no. 23, pp. 72-77. Junio 2018.

[7] Kapp, K. Game based methods and estrategies for training and education. New York: Pfeiffer. 2012, p. 67.

[8] Molina, M. "Uso de kits experimentales para mejorar las actitudes y bajar la repitencia en Química General". Revista Entre Ciencia e Ingeniería, vol. 12, no. 24, pp. 89-95. Diciembre 2018.

[9] Piaget, J. (2001). Sicología y Pedagogía. México: Edtorial Crítica. 2001, p. 38.

[10] Lopez, D., \& Mejía, L. "Una mirada a las estrategias y técnicas didácticas en la educación en ingeniería. Caso Ingeniería Industrial en Colombia”. Revista Entre Ciencia e Ingeniería, vol. 11, no. 11, pp. 123132. Junio 2017.

[11] Barriga, F., \& Hernandez, G. Estrategias docentes para un aprendizaje significativo: una interpretación constructivista. Ciudad de México: McGraw Hill Interamericana. 2002, p. 75.

[12] Pertuz, S., \& Torres, J. "Lineamientos para el diseño de Cursos Online Masivos y Abiertos (MOOC) en Ingeniería Electrónica". Revista Entre Ciencia e Ingeniería, vol. 11, no. 11, pp. 42-49. Junio 2017.

[13] Dooly, M. "Constructing knowledge together". En M. Dooly, Telecollaborative Language Learning. A guidebook to moderating intercultural collaboration online (págs. 21-45). Bern: Peter Lang. 2008.

[14] Adams, A. Cooperative learning effects on the classroom. Michigan: Northern Michigan University. Noviembre 2013, p. 51.

[15] Terenzini, P., Cabrera, A., Colbeck, C., Parente, J., \& Bjorklund, S. "Collaborative Learning vs. Lectura / Discussion: Students reporting learning gains". Journal of Engineering Education, pp. 123-130. Enero 2001.

[16] Blanchard, B. Ingeniería de Sistemas. Madrid (España): Isdefe. 2000, p. 78.

[17] Trejos, O. Significado y Competencias. Pereira: Papiro. 2013, p. 101.

[18] Lumsdaine, E., \& Lumsdaine, M. Creative Solving Problem: Thinking skills for a changing world. New York: McGraw Hill. 2005, p. 26.

[19] Herrmann, W. The whole brain. New York: McGraw Hill. 2000, p. 61.

[20] Rodas, A., Rios, J., \& Solarte, G. "Creación de una arquitectura utilizando Lenguaje de Modelado Unificado (UML) en la implementación de un Lenguaje Específico de Dominio Interno (LEDI)". Revista Entre Ciencia e Ingeniería, vol. 10, no. 20, pp. 15-23. Junio 2016.

[21] Van Roy, P. Techniques and methods in programming computer. Louvaine: University Press. 2008, p. 212.

[22] Trejos, O. I. Aprendizaje en Ingeniería: un problema de comunicación. Pereira (Colombia): Tesis Doctoral - Universidad Tecnológica de Pereira. 2012.

[23] Trejos, O. I. "Metodología de evaluación por conformación de grupos en un primer curso de programación de computadores usando aprendizaje Colaborativo". Revista Entre Ciencia e Ingeniería, vol. 12, no. 12 , pp. 58-65. Junio 2018

[24] Prince, M. "Does Active Learning work?" Journal Engineering Education, vol. 93, no. 3. 2004.

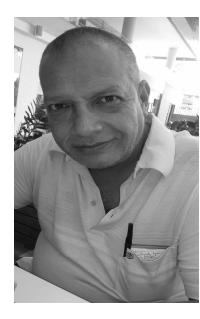

Omar Iván Trejos Buriticá es ingeniero de Sistemas, Especialista en Instrumentación Física, MSc en Comunicación Educativa, $\mathrm{PhD}$ en Ciencias de la Educación. Autor de varios libros de programación de computadores que se encuentran en el mercado. Sus artículos de investigación han sido publicados en revistas especializadas en Colombia y en el exterior. Ha dedicado su labor académica a investigar en diferentes estrategias para mejorar el proceso de aprendizaje de la programación de computadores de manera que se logre el objetivo de aprender por un camino expedito y duradero. Actualmente es docente de planta de la Universidad Tecnológica de Pereira, Facultad de Ingenierías, Programa de Ingeniería de Sistemas y Computación. ORCID: http://orcid.org/0000-0002-3751-6014. 